\title{
Infecção em Unidade de Terapia Intensiva: A auditoria Hospitalar na Prevenção e Controle
}

\author{
SILVA, Elieth Cruz Magno ${ }^{[1]}$, OLIVEIRA, Euzébio de ${ }^{[2]}$ \\ SILVA, Elieth Cruz Magno; OLIVEIRA, Euzébio de. Infecção em unidade de terapia intensiva: A \\ auditoria hospitalar na prevenção e controle. Revista Científica Multidisciplinar Núcleo do \\ Conhecimento. Ano 01, Ed. 08, Vol. 05, pp. 58-74, Agosto de 2016. ISSN: 2448-0959
}

\section{RESUMO}

A auditoria hospitalar trata-se de um ramo da contabilidade que tem sido usada por varias profissões, inclusive pela saúde, mais especificamente a enfermagem, nas organizações de saúde. A auditoria configura-se como uma importante ferramenta na transformação dos processos de trabalho que vem ocorrendo em hospitais, como o controle e prevenção de infecções, esse controle é feito pela Comissão de Controle de Infecção Hospitalar (CCIH) que possui como função realizar auditorias internas, avaliando o cumprimento do Programa de Controle de Infecção Hospitalar e minimização máxima da incidência e gravidade das infecções nosocomiais. As auditorias internas devem ser realizadas, periodicamente, pelas Unidades Hospitalares para observar o grau de infecção hospitalar presente. Objetivo: Analisar a funcionalidade de uma auditoria hospitalar na prevenção e controle de infecções em Unidade de Terapia Intensiva. Métodos: Serão coletados dados por meio de estudo bibliográfico no período de 2005 a 2014 nos seguintes bancos de dados: SCIELO (Scientific Eletronic Library) e LILACS (Literatura Latino Americana e do Caribe em Ciências da Saúde), Biblioteca da Universidade da Amazônia. A presente pesquisa é de caráter quantitativo. Conclusão: Ao término deste trabalho conluiu-se que apenas através de uma equipe de saúde intensivamente treinada e quanto às sugestões de cuidados universais é que será possível a prevenção das infecções hospitalares, sendo necessário que, todos os profissionais envolvidos respeitem e exerçam todas as normas estabelecidas pela CCIH. É importante ressaltar que as infecções hospitalares são adquiridas depois da admissão do paciente em um Hospital, que poderá aparecer durante a internação do paciente ou após sua alta e pode ser ocasionado devido alguns procedimentos hospitalares ou mesmo a internação.

Palavras-chaves: Auditoria Hospitalar. Prevenção. Infecção.

\section{INTRODUÇÃO}


Segundo Motta (2005), Riollino (2007) a palavra auditoria se origina do latim audire que significa ouvir. No entanto, o termo pode ser melhor esclarecido pela palavra da língua inglesa audit, o qual tem o significado de examinar, corrigir e certificar. Sendo assim, a auditoria incide na avaliação sistemática e formal de uma atividade para definir se ela esta sendo atingida de acordo com os seus objetivos. Trata-se de um ramo das ciências contábeis que tem sido usada por varias profissões, inclusive pela saúde, mais específico a enfermagem, devido a globalização e dada a necessidade das empresas de somarem o trabalho do auditor de enfermagem ao auditor médico, considerando sua função generalista. De acordo com Ribeiro (2009) atualmente, a auditoria em saúde se dispõe em algumas subdivisões que variam de sua classificação à sua execução.

Nas organizações de saúde, a auditoria configura-se como uma importante chave na transformação dos processos de trabalho que vem ocorrendo em hospitais, como o controle e prevenção de infecções (SCARPARO, 2007). A auditoria que condiz neste âmbito é a médica que consiste em uma avaliação dos aspectos qualitativos e quantitativos pautados na assistência em saúde. Também abrange aspectos organizacionais, operacionais e financeiros, com o objetivo de buscar qualidade dos cuidados prestados aos pacientes (SCARPARO, 2005). Atualmente é grande o número de profissionais que fazem auditoria em saúde e, para que seja executada de maneira correta, é necessário que os auditores (internos) tenham conhecimento das atividades que estão sendo auditadas, além do fluxo de auditoria e custos de materiais e medicamentos (REIS, 2008).

Segundo o Manual de Normas de Auditoria (1998) o Sistema Nacional de Auditoria - SNA, instituído pelo art. $6^{\circ}$ da Lei 8.689, de 27 de julho de 1993 e regulamentado pelo Decreto 1651, de 28 de setembro de 1995, abrange nos três níveis de gestão que compõem o SUS - Federal, Estadual e Municipal. Ele se reveste das atividades de auditoria, suplementando outras instâncias de controle e subsidiando o processo de planejamento das ações de saúde, sua execução, gerência técnica e avaliação qualitativa dos resultados obtidos.

Nesta visão, situa-se a elaboração do documento de orientação, conduzido aos auditores do SNA, ressalvando aspectos gerais e conceituais de auditoria, bem como o conjunto de ações técnicas e administrativas que compõem esta atividade. Tem por objetivo a aquisição da qualidade das ações e serviços prestados no campo da saúde, a ação de auditoria assinalar-se em atividades de averiguação analítica e operativa, incidindo no exame sistemático e independente de uma atividade, elemento ou sistema, para definir se as ações de saúde e seus resultados, estão de concordata com as disposições projetadas e com as normas e legislação vigentes (MANUAL DE NORMAS DE AUDITORIA, 1998).

A infecção hospitalar é toda infecção adquirida ou transmitida no espaço hospitalar. Atualmente, 15,5\% dos pacientes hospitalizados apresentam algum tipo de infecção. Esta condição alude altos custos para o Sistema Único de Saúde decorrente do aumento do tempo de internação e de cuidados terapêuticos e diagnósticos adicionais (MARTINEZ; CAMPOS; NOGUEIRA, 2008). Segundo Gastmeier (2009) essa situação está associada devido a muito tempo de internação, tanto em hospital, quanto em unidade de terapia intensiva.

Com o surgimento dos antibióticos, os médicos confiavam que as infecções desapareceriam; porém, o abuso por sua utilização, elegeu germes resistentes, aumentando o problema. A única forma de diminuir esse mal é pelo controle e a prevenção, coordenados por uma Comissão de Controle de Infecção 
Hospitalar, o que, embora seja uma exigência, apenas metade dos hospitais brasileiros possui essa comissão, e ainda assim uma minoria exerce atividades básicas de controle, de acordo com levantamento realizado pelo próprio Ministério da Saúde (CALVALLINI; BISSON, 2005). A Comissão de Controle de Infecção em Serviços de Saúde é um órgão que presta assessoramento a Direção Clínica da Unidade, tendo como aptidão a elaboração do Programa de Controle das Infecções Hospitalares, com a finalidade de favorecer a comunidade assessorada e protegerem o corpo clínico e o hospital (EVORA, 2008).

Portanto em 6 de janeiro de 1997 criou-se a Lei Federal 6.431, que obriga todos os hospitais brasileiros a constituírem Comissão de Controle de Infecção Hospitalar (CCIH) que necessitará agir de acordo com o programa desenvolvido pela própria instituição. A referida lei estabeleceu também a obrigação de instauração de um Programa de Controle de Infecção Hospitalar (PCIH), definido como um conjunto de ações desenvolvidas deliberada e sistematicamente com o objetivo de minimização máxima da incidência e gravidade das infecções nosocomiais (LUZ; MARTINS, 2007).

A comissão de controle de infecção hospitalar é formada por 2 níveis de performance, os consultores e os executores. Os consultores serão representados pelos serviços: médicos, enfermagem, farmácia e de microbiologia, os membros executores serão, no mínimo, 2 técnicos da área da saúde ou de nível superior para cada 200 leitos ou fração deste número com carga horária de 6 horas para o enfermeiro e 4 horas para os outros profissionais,sendo que 1 dos membros executores deve ser, um enfermeiro (ARAÚJO, 2009)

Sendo assim, o presente trabalho teve como objetivo analisar a funcionalidade de uma auditoria hospitalar na prevenção e controle de infecções em Unidade de Terapia Intensiva.

\section{METODOLOGIA}

Foram coletados dados por meio de estudo bibliográfico, sendo pesquisados 35 artigos no período de 2005 a 2014 nos seguintes bancos de dados: SCIELO (Scientific Eletronic Library) e LILACS (Literatura Latino Americana e do Caribe em Ciências da Saúde), Biblioteca da Universidade da Amazônia, para a pesquisa foi utilizado a palavra chave Auditoria, CCIH e Infecções. e detes, utilizados 22 mais relevantes, para a coleta de informações, A presente pesquisa é de caráter quantitativo.

\section{RESULTADOS E DISCUSSÃO}

\section{CONCEITUAÇÃO DE AUDITORIA MÉDICA}

O Instituto de Auditoria Interna do Brasil (AUDIBRA, 2011) afirma que a auditoria interna é um papel de avaliação independente, fundada dentro da organização para pesquisar e julgar suas atividades, como um serviço a essa mesma organização. O mesmo Instituto mostra, de modo claro, que do ponto de vista legal é função particular a auditoria da área contábil. Uma empresa de auditoria que se disponha, no Contrato Social, deixar clara tal imputação no objeto da empresa, ver-se-á obrigada a ter sócio-contador e registro no Conselho Regional de Contabilidade (CRC).

A auditoria médica é definida como o conjunto de atividades e ações de fiscalização, de controle e de avaliação dos processos e procedimentos adotados, assim como o atendimento prestado, objetivando sua melhor adequação e qualidade, detectando e saneando-lhes eventuais distorções e propondo medidas para 
seu melhor desempenho e resolubilidade. (CIAMPONE; KURCGANT, 2004). A definição de auditoria em saúde, segundo 64,3\% dos autores, está relacionada à análise qualitativa da assistência prestada, corroborando com a premissa do trabalho do profissional, que é oferecer ao seu cliente assistência de boa qualidade (SCARPARO, 2008).

Para medir a eficácia e a eficiência dos processos administrativos e assistenciais, podem-se utilizar práticas auditoras. A auditoria é exposta, discutida e determinada como um dos itens efetivos para a gestão da qualidade. Através dela, é possível detectar dificuldades, analisá-los e achar as causas que operam sobre as mesmas (MOTTA; LEAO; ZAGATTO, 2005).

O bom auditor deve ser um ilustre negociador nas várias situações, manifestando domínio de sua atividade. Finalmente, deve ser ético, respeitando seu código profissional e imparcial nas aplicações normativas, desempenhando de forma criteriosa, honesta e objetiva o seu trabalho, mantendo sigilo absoluto das informações confidenciais que chegarem a seu conhecimento (CHEBLI, 2007).

A auditoria médica pode ser alcançada de distintas formas, são elas: auditoria médica preventiva que é alcançada a fim de que os procedimentos sejam auditados antes que ocorram. Normalmente está unido ao setor de liberações de procedimentos ou guias do plano de saúde, e é desempenhada pelos médicos (GREGO, 2005; CIAMPONE, 2004). Na área da saúde, os profissionais que a desenvolvem devem ser capacitados especificamente para tal função (BRITO, 2006).

A auditoria médica operacional é o tempo no qual são auditados os procedimentos durante e após terem ocorrido $\mathrm{O}$ auditor age junto aos profissionais da assistência, com a finalidade de monitorizar o estado clínico do paciente internado, conferindo a procedência e gerenciando o internamento, ajudando na liberação de procedimentos ou materiais e medicamentos de alto custo, e também averiguando a qualidade da assistência prestada. É nesta hora que o auditor pode sugerir, com a anuência do médico assistente, outra alternativa de assistência médica ao usuário, como Gerenciamento de Casos Crônicos (GREGO, 2005; GAIDZINSKI, 2004).

Auditoria médica analítica abrange as atividades de exame dos dados levantados pela Auditoria Preventiva e Operacional, e da sua conferição com os indicadores gerenciais e com indicadores de outras organizações. Neste processo, os auditores devem ter conhecimento pertinente aos indicadores de saúde e administrativos, e no que fere a utilização de tabelas, gráficos, bancos de dados e contratos. Desta forma, são bons de reunir informações relacionadas ao plano de saúde, bem como quanto aos problemas detectados em cada prestador de serviços de saúde. Consequentemente, tais análises cooperam substancialmente para a gestão dos recursos da organização ou empresas públicas (BRANCO, 2001; ATTIE, 2005).

São diversas as formas e modalidades de auditoria que existem em saúde, que direcionam o trabalho para uma especificidade, mas de forma geral, todas estabelecem que o auditor seja responsável em sua atuação (MELO, 2008).

A atividade de auditoria médica está mencionada no rol das funções do médico, por meio da RESOLUÇÃO CFM n 1.627/2001: “As atividades de coordenação, direção, chefia, perícia, auditoria, supervisão e ensino dos procedimentos médicos privativos incluem-se entre os atos médicos e devem ser exercidos unicamente por médico". 
Mesmo as resoluções que definem as especialidades médicas, como a RESOLUÇÃO CFM ${ }^{\circ}$ 1.763/2005, deixam a questão da auditoria para outra instância, conforme o texto: "Auditoria será designada área de atuação especial e receberá outro tipo de 3 especificação".

Inclui-se na auditoria médica operacional a auditoria de contas - rotulada como visita hospitalar de alta que acontece depois a alta hospitalar do paciente. Porém, ainda no ambiente hospitalar, tal processo advém antes desta conta ser mandada para a fonte pagadora, tendo o auditor o direito do prontuário médico completo para análise. Neste caso, possíveis irregularidades ou inconformidades podem ser negociadas antes do envio da conta hospitalar à fonte pagadora, com mútua e formal concordância. A mais uma possibilidade é da auditoria de contas ser atingida nas instalações da organização pagadora. $\mathrm{Na}$ auditoria de contas encontram-se os padrões das Comissões de Controle de Infecção Hospitalares (CCIH) (KOTLER, 2005; FALK, 2001).

Auditoria, no entanto, é uma função administrativa de aplicação primordial em toda a engrenagem/sistemática/mecânica empresarial compondo com as funções planejamento, implemento, domínio do ciclo administrativo, ampliação da qualidade a ser praticada por todos os profissionais complementares/ partícipes das organizações (SANTOS, 2008; TIPPLE, 2003).

$\mathrm{Na}$ área da saúde, acabou surgindo a auditoria hospitalar que vem com novas dimensões, manifestando sua importância dentro das instituições hospitalares. Trata-se de uma avaliação sistemática e formal de uma atividade que é desenvolvida por um profissional, subsidiando a qualidade do atendimento apresentado, favorecendo clientes, famílias e profissionais. Ao aplicar a técnica de auditoria, identificando-se e solucionando os problemas, pode-se garantir a administração da qualidade assistencial (ALMEIDA, SANTOS 2008).

Quando o serviço de auditoria for implementado na instituição hospitalar, ela estará interligando os demais departamentos, e essa interligação permitirá o sucesso deste trabalho (CARNEIRO, 2009; SANTOS, 2008).

\section{COMISSÃO DE CONTROLE DE INFECÇÃO HOSPITALAR}

A história da infecção hospitalar está implantada na história da medicina que começou com a luta pela sobrevivência e até por um mundo claramente governado por forças poderosas e ocultas. Por séculos a humanidade usou de medicina arcaica, porém, com a demonstração e a observação, especialmente na Grécia, Índia e China o conhecimento foi se explanando até a medicina se firmar como ciência (OLIVEIRA, 2005; CORREA, 2008).

Somente em 460 a.C. que Hipócrates, registrou os fenômenos biológicos, colaborou para que se cuidasse das feridas, destacou a importância da lavagem de mãos anteriormente a cirurgias, o uso de água fervida e vinho para a limpeza dos ferimentos. Posteriormente a Hipócrates, Galeno, um médico grego, relatou a cura sem a formação de secreções em feridas de gladiadores após a lavagem com vinho e o fechamento com fios de linho (FONTANA, 2006).

A partir da primeira metade o século XIX é que a infecção hospitalar principiou a ser focalizada por profissionais da saúde. Relatos mostram que em 1847, o médico Ignaz Phiipp Semmelweis, em Viena, preconizou a lavagem de mãos com água clorada para todo o examinador, antes de tocar a parturiente. 
Com esta medida pode-se diminuir drasticamente a taxa de mortalidade materna por febre puerperal (FONTANA, 2006; TURRINI, 2004).

Contudo, o começo das preocupações com as infecções hospitalares no Brasil seguiu o processo de industrialização rápido que se principiou no governo de Juscelino Kubischek e com o acontecimento de surtos por estafilococo resistente a penicilina, pelos relatos vindos de outros países com medicina tecnologicamente avançada (ANVISA, 2000).

A década de 80 foi a mais respeitável até a ocasião para o desenvolvimento do controle de infecção hospitalar no Brasil. Principiou a ocorrer uma conscientização de profissionais da saúde à importância do tema e constituíram diversas comissões de controle nos hospitais. Neste contexto, a Agência Nacional de Vigilância Sanitária (ANVISA) é responsável pela coordenação do Programa Nacional de Controle de Infecção Hospitalar (PNCIH). O PNCIH ordena ações nacionais de prevenção e controle de infecção hospitalar, estabelecendo critérios, parâmetros e métodos para o desenvolvimento das atividades (ARAÚJO, 2009; GIUNTA, 2006).

A fundação desses programas é muito importante porque as infecções hospitalares comumente colaboram para aumentar a duração hospitalar do paciente e a utilização de recursos dispendiosos para o seu tratamento, permitem ações legais contra o profissional e o hospital e envolvem a imagem da organização diante dos consumidores (GONTIJO, 2006; GIUNTA, 2006).

Para um eficaz controle de infecção hospitalar é preciso ter no hospital

profissionais motivados, trabalhando em equipe, respeitando cada um dentro de suas funções, atualizandose com frequência e com capacidade de auto avaliarem-se (BRITO, 2006).

Em 6 de janeiro de 1997, fundou-se a Lei Federal 6.431, a qual estabelece a obrigatoriedade da existência de uma Comissão de Controle de Infecção Hospitalar (CCIH) e de um Programa de Controle de Infecção Hospitalar (PCIH), determinado como um conjunto de ações, desenvolvidas, deliberada e sistematicamente, com o objetivo de diminuir, ao máximo possível, a incidência e a gravidade das infecções (FERRAZ, 2010; PINHEIRO, 2008).

A CCIH deverá ser composta por profissionais da saúde, de nível superior, designados; serão divididos em dois tipos: consultores e executores, sendo os consultores emissários dos seguintes serviços: médico, enfermagem, farmácia e microbiologia; e os membros executores da CCIH representantes do SCIH, e, portanto, são incumbidos da efetivação de ações programadas para o controle (SEQUEIRA, 2005; PEREIRA, 2005).

Segundo a Secretaria do Estado de Góias (2010) as finalidades da CCIH são: referendar um sistema de vigilância epidemiológica; aferir normas técnicas integrantes, de acordo com as particulares da maternidade, para a prevenção de infecções, com destaque na regulamentação das obrigações e medidas de isolamento e acompanhamento de sua atenção; recomendar medidas que procedam na prevenção ou diminuição de infecções hospitalar; implantar, através do SCIH, o controle do uso de antimicrobianos; noticiar o Regimento Interno, suas atribuições, finalidades e objetivos, mirando o entendimento e a cooperação de todos os servidores da Instituição. 
Outras finalidades são: inserir um sistema de informação e prevenção de doenças endêmicas que possam contribuir para suavizar os índices de infecção hospitalar; notificar programas de atualização pelo intercâmbio de informação e experiências, com outras Instituições, no âmbito municipal, estadual, nacional e internacional; incentivar e contribuir, no âmbito de sua competência, para a pesquisa e a implantação de metodologias racionais que possam contribuir para a dinamização dos controles de infecção hospitalar; padronização e rotinas para uso dos produtos e métodos de controle de qualidade dos germicidas, inseticidas, antissépticos e saneantes (SECRETARIA DO ESTADO DE GÓIAS, 2010).

O Controle de Infecção Hospitalar (CIH) é um órgão executivo, ligado à Vice-Presidência Médica encarregado do cumprimento das ações planejadas de prevenção e domínio de infecções hospitalares admitidas pela CCIH (HCPA, 2010).

Conforme descreve a Portaria 2.616, de 1998, é competência da CCIH a realizar auditorias internas avaliando o cumprimento do Programa de Controle de Infecção Hospitalar e registrá-los. As auditorias internas devem ser realizadas, periodicamente, pelas Unidades Hospitalares, através de protocolos específicos para verificar o cumprimento da legislação específica que trata do Controle de Infecção Hospitalar.

A função da auditoria na $\mathrm{CCIH}$ é identificar problemas na estrutura, no processo e na consequência, ministrando informações que aprovem ações que aprimorem a qualidade da assistência. A auditoria hospitalar é unidade administrativa que tem a competência de ajudar os gestores na execução da missão e na continuidade da entidade, como resultado, habilidade de inovar, fazendo mais e melhor com menos recursos, chegando a metas previamente definidas e agregação de pessoas cada vez mais satisfeitas com o padrão de serviços apresentados (BRITO; FERREIRA, 2006)

A inexistência de normalização e socialização das diversas práticas são comumente verificadas e provocam na ineficiência do serviço de organizar as normas e rotinas (Procedimentos Operacionais Padrão - POP) para o seu funcionamento, que devem ser adotadas uniformemente por todos os profissionais. Sua preparação pode ser feita em parceria com o Serviço de Educação Continuada e a Comissão de Controle de Infecção Hospitalar, seguido de capacitação para a socialização das informações instituídas no serviço aos profissionais de saúde (PEREIRA, 2005; FERNANDES, 2000).

A inatividade ou ineficiência da Comissão de Controle de Infecção Hospitalar (CCIH) é outro fato muito comum encontrado nos hospitais e que acaba ocasionando prejuízos à instituição, seja pela falta de capacidade ou perfil dos profissionais para desempenhar na área ou por falta de conscientização do gestor quanto às necessidades sugeridas pela $\mathrm{CCIH}$, nem sempre atendidas. Muitas vezes ocorre da $\mathrm{CCIH}$ não dispor de um Programa de Controle de Infecção Hospitalar (PCIH) implantado e implementado, trabalhando sem um direcionamento para suas ações (SILVA, 2005; ALVES, 2002).

Muitos hospitais ainda não atentaram para a importância da contribuição da CCIH na assessoria administrativa da instituição, diagnosticando e atentando a assiduidade e distribuição das infecções hospitalares entre os pacientes internados e egressos, interferindo através da implantação e/ou implementação de medidas de controle de infecção, visando garantir a qualidade e segurança da sua assistência (SCARPARO, 2005; BRITO, 2006). 
A preocupação em sustentar o controle das infecções hospitalares no Brasil apareceu na década de 60, nascendo também às primeiras publicações e relatos arrolados ao tema (ROCHA; LEME, 2010).

São diversos fatores que aumentam os números de casos de infecção na UTI. Podemos mencionar aqueles relacionados ao paciente como: transplantes, imunodepressão, queimaduras, procedimentos e técnicas aos quais os pacientes estão exibidos (LICHY; MARQUES, 2007).

As infecções hospitalares são os indicativos mais evidentes da inconformidade do sistema de saúde, embora a responsabilidade seja, em geral, conferida ao profissional de saúde ou à instituição prestadora de assistência (OLIVEIRA, 2005).

O controle de infecções em UTI é um assunto difícil e de grande importância para o adequado funcionamento da unidade, evidenciando-se, portanto, a precisão da proteção tanto individual quanto dos pacientes, bem como a concretização de técnicas e procedimentos apropriadas a fim de impedir qualquer prejuízo para o paciente, outro fator, não menos importante é a limpeza do ambiente hospitalar (PINHEIRO; NICOLETTI, 2008). Assim sendo, a higienização das mãos no ambiente hospitalar, passa a ser uma prática prioritária, avaliando ser o isoladamente mais importante para reduzir as taxas dessas infecções no ambiente hospitalar, por diminuir a transmissão de microorganismos pelas mãos (LIRA, 2008)

É de extrema importância salientar a necessidade de uma CCIH dentro da Unidade Hospitalar e principalmente dentro da Unidade de Terapia Intensiva. Assim também assumir o quanto importante é a participação do enfermeiro, para que tenha um apropriado desempenho diante às dificuldades apresentadas causadas pela imprudência, imperícia e até mesmo falta de informação de alguns profissionais deste ambiente (PEREIRA; SOUZA, 2005).

O trabalho de uma equipe multidisciplinar de auditores tende a alcançar maior sucesso nos resultados do que os trabalhos desenvolvidos somente por uma categoria profissional, pois cada uma delas audita e avalia melhor os procedimentos de sua área de formação específica (REIS, 2007).

A CCIH traz o objetivo não unicamente de prevenir e combater à infecção hospitalar, favorecendo dessa maneira toda a comunidade assistida, como também de proteger o hospital e o corpo clínico. Em uma Unidade de saúde, a CCIH é uma base a todos os profissionais que operam diretamente com os pacientes com a finalidade de prevenir e controlar as infecções hospitalares e suas prováveis conseqüências (CORRÊA, 2008; CAVALLINI, 2005).

As CCIHs das instituições têm um papel importantíssimo, para analisar e apurar o causador ou culpado em casos de IH (ANTÔNIO, 2000).

A aniquilação da IH é tarefa impossível, sendo que sua etiologia e condições de instalação no homem em desequilíbrio no seu processo doença. Portanto, a profilaxia e redução têm se mostrado viáveis em vários casos e situações, conforme já comprovado na prática hospitalar (CORRÊA, 2008; CAVALLINI, 2005). As CCIHs trabalham com uma política de Vigilância Epidemiológica de IH definida, ligadas a saúde do trabalhador e a prevenção de risco ocupacional (MOSCOVICI, 2007). 
A criação e o funcionamento das $\mathrm{CCIH}$ concebem um progresso na organização da estrutura hospitalar para a redução de múltiplos problemas, como a necessidade de se diminuir e controlar taxas de infecções, o que determinou a aplicação de medidas preventivas, educacionais e de controle epidemiológico, que tendem, através de um processo de conscientização coletiva, a levar as taxas de infecção para limites aceitáveis (ZAVASCKI; CRUZ, 2005).

Portanto, é necessário que as CCIH estudem e determinem, de forma coletiva e institucional, as ações de vigilância, de treinamento e de medidas burocráticas (controle e encaminhamento) que contemplem os profissionais da saúde e todos aqueles que fazem a assistência hospitalar (ODELET, 2007).

A respeito da forte evidência da efetividade e eficácia das atividades de vigilância e controle de infecção, não há estudo que evidencie, precisamente, quais métodos e programas devem ser tomados, na execução da vigilância e controle de infecção hospitalar. A modificação de comportamento, tão necessária neste contexto, não incide em passo acelerado. É preciso a fundamentação prática e teórica e, ainda, a absorção e aplicação de medidas preventivas, imperativas à adequada assistência. A formação de hábitos pelos profissionais e, não apenas, a teorização do conhecimento, toma-se em objetivo a ser alcançado pela CCIH (PITET, 2005; PUCCINI, 2008).

Assim, a problemática das infecções hospitalares requer também alterações de ordem governamental, como a concepção de uma política para o controle de infecção de máxima efetividade, que vá além do estabelecimento de mecanismos legais e normativos para a sua regulação, que abarque a população usuária dos serviços, tornando-a partícipe no processo (TURRINI, 2002; PUCCINI, 2008).

É preciso refletir sobre todas as estratégicas aceitáveis que possam colaborar para a mudança do atual panorama que se expõe, como: a introdução da temática nos currículos dos cursos de graduação na área de saúde; o aumento dos investimentos na disponibilização de cursos de pós-graduação em controle de infecção para profissionais de saúde, especialmente fora dos grandes centros; garantias legais no reconhecimento e autonomia do profissional controlador de infecção; revisão da legislação vigente objetivando o cumprimento efetivo das medidas de prevenção e controle, tanto para estabelecimentos públicos como privados; investimentos em pesquisas, seminários e atualizações (COUTO, 2003; LENTZ, 2003).

Assim, a área de auditoria em saúde no Brasil constitui um campo aberto a novos estudos, pesquisas e registros que possam incrementar a prática da atividade, que vem adquirindo cada vez mais importância diante dos custos crescentes na área da saúde (REIS, 2007).

\section{CONCLUSÃO}

Ao término deste trabalho conclui-se que apenas através de uma equipe de saúde intensivamente treinada quanto às sugestões de cuidados universais é que será provável a prevenção das infecções hospitalares, sendo necessário que, todos os profissionais respeitem e exerçam todas as normas estabelecidas pela $\mathrm{CCIH}$.

A Comissão de Controle de Infecção Hospitalar (CCIH) é constituída por um grupo de profissionais que têm funções de prevenção de infecções hospitalares, de sustentar e avaliar o Programa de Controle de Infecção Hospitalar (PCIH), entre outras funções. 
É importante ressaltar que as infecções hospitalares são adquiridas depois da admissão do paciente em um Hospital que poderá aparecer durante a internação do paciente ou após sua alta e pode ser ocasionada devido alguns procedimentos hospitalares ou mesmo a internação.

A realidade social do desenvolvimento do modo de produção dominante de assistência à saúde vem exigindo novos processos de trabalho não relacionados com o cuidado direto ao paciente, sendo diversos deles realizados por enfermeiros como: $\mathrm{CIH}$, controle de qualidade hospitalar, como também o gerenciamento da higiene hospitalar.

\section{REFERÊNCIAS}

ADELINO F.O. Auditoria interna e controle governamental. Tribunal de Contas da União. Brasília: 2011.

AGÊNCIA NACIOAL DE VIGILÂNCIA SANITÁRIA (ANVISA). Curso Básico de

Infecção Hospitalar. 2000.

ALMEIDA, E. R. D. Auditoria Hospitalar: auditoria interna em saúde. Campinas, 2008.

ALVES, D.C.I; ÉVORA, Y.D.M. Questões éticas envolvidas na prática profissional de enfermeiros da comissão de controle de infecção hospitalar. Rev Latino-Am Enferm , 265-75, 2002.

ANTÔNIO, L.A.C. Aspectos legais no Controle de Infecção Hospitalar. In: Fernandes AT, Fernandes MOV, Ribeiro Filho, N. Infecção Hospitalar e suas Interfaces na área da Saúde. $1^{a}$ ed. São Paulo: Atheneu; 2000.

ARAÚJO, R.Q. Participação da Farmácia Hospitalar no Controle das Infecções Hospitalares. Revista Racine. Rio de Janeiro: 2009. p50-59.

ATTIE, W. Auditoria: Conceitos e Aplicações. São Paulo : Atlas, 2005

BRANCO, M.A.F. Informação em saúde como elemento estratégico para gestão. in: MS, Gestão municipal da saúde, textos básicos. Rio de Janeiro: Brasil, Ministério da Saúde, 2001, p. 163-169

BRITO M.F, FERREIRA L.N. A importância da auditoria interna hospitalar na gestão estratégica dos custos hospitalares. Ciências Contábeis da Universidade Católica de Brasília. Trabalho de conclusão de curso. 2006

CARNEIRO, R. M.; OLIVEIRA, S. M. D.; NAKAMURA, E. K. Educação Continuada para a Enfermagem. Disponível em: www.uniandrade.edu.br/links/menu3/publicações/revistaenfermagem/oitavo-amanha/artigo14.pdf. Acesso em: 07 de setembro 2015.

CAVALLINI, M.E; BISSON, M.P. Farmácia Hospitalar. Um enfoque em sistemas de saúde. Barueri: Manole, 2005. p170. 
CIAMPONE, M. H. T.; KURCGANT, P. O Ensino de Administração em Enfermagem no Brasil: o processo de construção de competências gerenciais. Revista Brasileira de Enfermagem. Brasília (DF): p.401-407, 2004.

COUTO, R. C.; PEDROSA, T. M. G.; NOGUEIRA, J. M. Infecção hospitalar: epidemiologia, controle e tratamento. 3. ed. Rio de Janeiro: Médica e Científica, 2003.

COUTO, R.C; PEDROSO, E.R.P; PEDROSA T.M.G. História do Controle da Infecção Hospitalar no Brasil e no Mundo. In: Couto RC, Pedrosa TMG, Nogueira JM. Infecção Hospitalar e outras Complicações Não-infecciosas da Doença. $3^{\text {a }}$ edição. Rio de Janeiro: Medsi; 2003.

CORRÊA L. Impacto da prevenção das infecções relacionadas à assistência a saúde: segurança e redução de custos. Einstein: Educ Contin Saúde 2008, p 194-196.

CHEBLI, T.F; MAIA, J.R; PAES, P.P.L. Manual de auditoria de contas médicas. Ministério da Defesa, Exército Brasileiro, $4^{\circ}$ Região Militar, 4 Divisão do Exército, Juiz de Fora - MG, 2007.

ÉVORA, Y.D.M; ALVES. D.C.I. Questões éticas envolvidas na prática profissional de enfermeiros da comissão de controle de infecção hospitalar. Revista Latino-Americana de Enfermagem, 265-275, 2008.

FALK, J. A. Gestão de custos para hospitais. São Paulo: Atlas, 2001.

FERNANDES, A.T; FERNANDES, M.O.V. Organização e Programa do Controle das Infecções Hospitalares. In: FERNANDES, A.T. Infecção hospitalar e suas interfaces na área da saúde. São Paulo: Atheneu; 2000.

FERRAZ, E.M, et al. Controle de infecção em cirurgia geral - Resultado de um estudo prospectivo de 23 anos e 42274 cirurgias.Revista do Colégio Brasileiro de Cirurgiões: 2010.

FONTANA, R.T. As infecções hospitalares e a evolução histórica das infecções. Revista Brasileira de Enfermagem. 2006, p 703-6.

GASTMEIER, P; SOHR, D; GEFFERS, C; RUDEN, H; VONBERG, R.P; WELTE, T. Early-and late -on set pneumonia: Is this still a useful classification? Antimicrob Agents Chemother, 2714-8, 2009.

GAIDZINSKI, R. R.; PERES, H. H. C.; FERNANDES, M .F. P. Liderança: aprendizado contínuo no gerenciamento em Enfermagem. Revista Brasileira de Enfermagem. Brasília (DF): p.464-466, 2004.

GIUNTA, A. P. N.; LACERDA, R. A. Inspeção dos programas de controle de infecção hospitalar dos serviços da saúde pela vigilância sanitária: diagnóstico de situação. Revista da Escola de Enfermagem. São Paulo. 2006. p. 64-70.

GRECO, R.M. Ensinando a Administração em Enfermagem através da Educação em Saúde. Brasília (DF). Revista Brasileira de Enfermagem. 2005: p.504-507,.

GONTIJO FILHO, P.P. Problemas da vigilância epidemiológica de infecções hospitalares sem o uso de 
critérios microbiológicos no Brasil. Revista de Ciências Farmacêuticas Básica e Aplicada. 2006.

HOSPITAL DE CLÍNICAS DE PORTO ALEGRE. Comissão de Controle de Infecção Hospitalar. Porto Alegre: Portal Web do HCPA, 2010. Disponível em: htpp//:www.hcpa.ufrgs.br/content/view/1123/1068. Acesso em 18 jun 2014.

Instituto de Auditoria Interna do Brasil. Papel da auditoria interna. Disponível em: <http//: http://www.iiabrasil.org.br/new/>acesso em 17 junho 2014.

JODELET, D. Imbricações entre Representações Sociais e Intervenção. In: MOREIRA, A.S.P.M; CAMARGO, B.V. Contribuições para a teoria e o método de estudo das Representações Sociais. João Pessoa (PB): Ed. Universitária. 2007.

KOTLER, P. Administração de Marketing. Prentice-Hall, 13 ${ }^{a}$ Edição, São Paulo: 2005.

LENTZ, R.A; NASCIMENTO, K.C; KLOCK, P. Infecções hospitalares: um desafio aos profissionais de saúde. In: ERDMANN, A.L; LENTZ, R.A; organizadoras. Aprendizagem continua no trabalho: possibilidades de novas práticas de controle de infecções hospitalares. São José: SOCEPRO; 2003

LICHY; R.Fá., MARQUES; I.R. Fatores de Risco para Infecção Hospitalar em Unidades de Terapia Intensiva: Atualização e Implicações para a Enfermagem. Rev Enferm. UNISA 2007; p 43.

LIRA, M.C; HINRICHSEN, S.L; ANJOS, A.B; BORGES, M.G.A; PEREIRA, M.G.B; DANDA, G.J.N. Higienização das mãos. In: HINRICHSEN, S.L. Biossegurança e Controle de Infecções - risco sanitário hospitalar. Rio de Janeiro (RJ): MDSI; 2008

LUZ A, MARTINS AP, DYNEWICZ AM. Caracteristicas de anotações de enfermagem encontradas em auditoria. Rev. Eletrônica Enferm. Disponivel em: http://www.fen.ufg.

br/revista/v9/n2/v9n2a05.htm.

Manual de Normas de Auditoria. Auditoria Médica. Diretora, Deildes de Oliveira Prado et all. - Brasília: Ministério da Saúde, 1998.

MARTINEZ, M.R.; CAMPOS L.A.A.F.; NOGUEIRA C.P.K. Adesão a técnica de lavagem de mãos em Unidade de Terapia Intensiva Neonatal. Revista Paulista de Pediatria. 2008, p179-85.

MElo, M.B; VAITSMAN, J. Auditoria e avaliação no Sistema Único de Saúde. Rev São Paulo Persp.,152-62,2008

MOTTA A.L.C, LEAO E., ZAGATTO J.R. Auditoria Medica no Sistema Privado: abordagem pratica para organizacoes de saude. Sao Paulo: Iatria, 2005.

MOSCOVICI, S. A relatividade tem 100 anos. In: MOREIRA, A.S.P; CAMARGO, B.V. Contribuições para a teoria e o método de estudos das Representações Sociais. João Pessoa (PB): Ed. Universitária, 2007. 
OLIVEIRA, A.C. et al. Infecções hospitalares: Epidemiologia, prevenção e controle. Rio de Janeiro: Guanabara, 2005.

PEREIRA M.S, SOUZA A.C.S. Tipple AFV, Prado MA. A infecção hospitalar e suas implicações para o cuidar da enfermagem. Texto Contexto Enfermagem: 2005. p 250-257.

PITTET, D. Infection control and quality heath care in the new millennium. American Journal Infection Control, St. Louis, v. 33, p. 258-267, 2005.

PINHEIRO, M.S., NICOLETTI, C.; BOSZCZOWSK, I.;PUCCINI, D.M. RAMOS, S.R.: Infecção hospitalar em Unidade de Terapia Intensiva Neonatal: há influência do local de nascimento. Rev Paul Pediatria. 2008; p6-14.

REIS,A.; SILVA, L.S. A História da Contabilidade no Brasil. Rev UNIFACS, 25-9, 2007.

RIBEIRO, L. Auditoria em Saúde. In: Especialização em Auditoria e Gestão em Saúde. Curitiba: Universidade Tuiuti, aula, 2009.

RIOLINO N.A, KLIUKAS G.B.V. Relato de Experiencia de Enfermagem no campo de Auditoria de Prontuario - uma acao inovadora. Sao Paulo: Nursing, 2003. p 35-38.

ROCHA; L.F., LEME N.A.; BRASILEIRO; M.E.: A Atuação da Comissão de Controle de Infecção em Serviços de Saúde na Unidade de Terapia Intensiva: O que fazer?. Revista Eletrônica de Enfermagem do Centro de Estudos de Enfermagem e Nutrição. [Serial online] 2010 Jan-Jul 1 (1) 1-16. Available from:http://www.ceen.com.br/revistaeletrônica.

SANTOS, C. E. T. D. A Relevância do Enfermeiro na Auditoria de Contas Médicas. [Trabalho de Conclusão do Curso de Pós-Graduação]. Campinas SP; 2008.

SECRETARIA DE SAÚDE DO ESTADO DE GOIÁS. Comissões. Disponível em:<http://www.saude.go.gov.br/index.php?idEditoria=4395>Acesso em: 18 junho 2014.

SEQUEIRA, E. J.D. Biossegurança. Rio de Janeiro: Medsi, 2005.

SILVA, Z.P; COROA, M.L. Vigilância Sanitária: um histórico discursivo. REVISA. 2005.

SCARPARO A.F. Auditoria de Enfermagem - identificando sua concepcao e metodos. [dissertacao]. Ribeirao Preto (SP): Escola de Enfermagem de Ribeirao Preto, Universidade de Sao Paulo; 2007.

SCARPARO AF. Auditoria em Enfermagem: revisao de literatura. Nursing. Sao Paulo: p46-50.2005.

SCARPARO, A.F; FERRAZ, C.A. Auditoria em Enfermagem: identificando sua concepção e métodos. Rev Bras Enferm.34-40,2008.

TIPPLE, A.F.V; PEREIRA, M.S; HAYASHIDA, M; MORIYA, T.M; SOUZA, A.C.S. O ensino do controle de infecção: um ensaio teóricoprático. Rev Latino-Am Enferm. 245-50, 2003. 
TURRINI, R.N.T; SANTO, A.H. Infecção Hospitalar e causas múltiplas de morte. J. Pediatr. (Rio de Janeiro). 2002

Turrini R.N.T. Programa de controle de infecção hospitalar: problemas na implementação em hopsitais do município de São Paulo. Acta Paul. Enf. 2004; p.316-324.

VAGHETTI, H. et al. Percepções dos enfermeiros acerca das ações administrativas em seu processo de trabalho. Brasília (DF). Revista Brasileira de Enfermagem. 2006, p.316- 320.

ZAVASCKI, A.P.; CRUZ, R.P.; GOLDANI, L.Z. Risk factors for imipenem-resistant Pseudomonas aeruginosa: a comparative analysis of two case-control studies in hospitalized patients. Infection control and hospital epidemiology, Thorofare, 2005. p. 96-101.

${ }^{[1]}$ Graduanda em Fisioterapia pela Universidade Federal do Pará.

${ }^{[2]}$ Biólogo, Mestre em Biologia. Doutor em Medicina/Doenças Tropicais. Professor e Pesquisador em na Universidade Federal do Pará - UFPA. E Pesquisador Colaborador no Núcleo de Medicina Tropical da UFPA.

\section{PUBLIQUE SEU ARTIGO CIENTÍFICO EM:}

https://www.nucleodoconhecimento.com.br/enviar-artigo-cientifico-para-submissao 